\title{
THE MAY MEETING IN YOSEMITE
}

The five hundred third meeting of the American Mathematical Society was held at Yosemite National Park on Saturday, May 1, 1954. Attendance was approximately 125 , including 82 members of the Society.

All meetings were held in the office and recreation room of Camp Curry. By invitation of the Committee to Select Hour Speakers for Far Western Sectional Meetings, Professor P. A. White of the University of Southern California presented an hour address entitled Regular convergence. Professor White was introduced by Professor J. L. Kelley. The four sessions for contributed papers were presided over by Professors C. B. Morrey, Ivan Niven, Roy Dubisch, and Leon Henkin.

Abstracts of papers presented at the meeting follow. Mr. Maschler and Dr. Bremermann were introduced by Professor Stefan Bergman, Mr. Miller by Professor R. H. Sorgenfrey, and Dr. Fraissé by Professor Alfred Tarski. Abstracts whose numbers are followed by the letter " $t$ " were presented by title. In the case of joint papers, (p) following one of the authors' names indicates the one who actually read the paper.

\section{Algebra ANd Theory of Numbers}

548. S. P. Avann: Dual symmetry of projective sets in a finite modular lattice.

Let $Q$ be any complete set of projective complemented quotient sublattices of a finite modular lattice $L$. Let $V_{Q, k}(L)$ be the set of elements $x$ for which there are precisely $k$ quotients in $Q$ that are minimal in the complemented quotient lattice $x^{*} / x$, generated above $x$ by the elements covering $x$. Define $W_{Q, k}(L)$ dually. The orders of $V_{Q, k}(L)$ and $W_{Q, k}(L)$ are equal. For $k=1$, the number of maximal quotients of $Q$ equals the number of minimal quotients of $Q$. When the orders of $V_{Q, k}$ and $W_{Q, k}$ are summed over all complete sets of projective prime (two-element) quotients, one obtains a recent result of Dilworth. The number of elements of $L$ covered by precisely $k$ elements equals the number of elements of $L$ covering precisely $k$ elements. If, furthermore, $k=1$, the number of meet-irreducibles and the number of join-irreducibles in $L$ are equal. The abstract Moebius function is employed to obtain the results. (Received March 10, 1954.)

\section{9t. J. L. Brenner: The direct product of simple groups.}

Let $G_{n}$ be the simple group $S L_{4 n}[F]$, and let $G$ be the direct product of the $G_{n}$ taken in order. $G$ has certain obvious normal subgroups which arise from the general properties of the direct product. It is shown that $G$ has still other normal subgroups, and all the normal subgroups of $G$ are found. (Received January 18, 1954.) 


\section{Chen-Chung Chang and Anne C. Davis (p): Some theorems on ordinal products of relations.}

The ordinal product $R \cdot S$ of binary relations $R$ and $S$ is the set of ordered pairs $\left\langle\langle r, s\rangle,\left\langle r^{\prime}, s^{\prime}\right\rangle\right\rangle$ where $r, r^{\prime}$ belong to the field of $R, s, s^{\prime}$ to the field of $S$, and either (i) $s \neq s^{\prime}$ and $\left\langle s, s^{\prime}\right\rangle \in S$ or (ii) $s=s^{\prime}$ and $\left\langle r, r^{\prime}\right\rangle \in R$. (Related definitions are found in Whitehead and Russell, Principia Mathematica, vol. II, 2d ed., 1927, p. 310.) If $\alpha, \beta$ are the (isomorphism) types of relations $R, S$ respectively, then the type of the relation $R \cdot S$ is denoted by $\alpha \cdot \beta$, the power of the field of the relation $R$ by $\mathfrak{p}(\alpha)$. Let $\alpha^{1}=\alpha$, $\alpha^{n+1}=\alpha^{n} \cdot \alpha$ for every natural $n$. The following theorems hold for types of nonempty reflexive relations. I. If $\alpha \cdot \gamma=\beta \cdot \delta$ and if $\mathfrak{p}(\alpha)=p(\beta)<\mathcal{N}_{0}$, then $\gamma=\delta$. An immediate consequence of I is: II. If $\alpha^{n}=\beta^{n}$ and if $p(\alpha)<\aleph_{0}$, then $\alpha=\beta$. III. If $\alpha \cdot \gamma=\beta \cdot \gamma$, $\mathfrak{p}(\alpha)<\boldsymbol{N}_{0}, \mathfrak{p}(\gamma)<\boldsymbol{\aleph}_{0}$, then $\alpha=\beta$. From I and III one easily obtains: IV. If $\alpha \cdot \beta=\gamma \cdot \delta$, $\mathfrak{p}(\alpha)<\boldsymbol{\aleph}_{0}, \mathfrak{p}(\beta)<\boldsymbol{\aleph}_{0}$, and if either $\mathfrak{p}(\alpha)=\mathfrak{p}(\gamma)$ or $\mathfrak{p}(\beta)=\mathfrak{p}(\delta)$, then $\alpha=\gamma$ and $\beta=\delta$. (Received March 10, 1954.)

\section{C. J. Clark: Introduction to $C-R$ algebras.}

This paper introduces the concept of closure-relation (C-R) algebras described by a collection of axioms which prove to be satisfied if one takes the elements of the algebra to be the set of all binary relations between elements of a compact first countable Hausdorff space, and the fundamental operations of this algebra to be the operations of join, meet, product, converse, and closure. This abstract algebraic system, which is an extension of the notion of closure algebra, is a Boolean algebra with operators. In this system the closure operation is extended from elements of a Boolean algebra to those of a relation algebra. This necessitates additional axioms on operations and elements peculiar to relation algebras. Definitions are given for the operations of taking the domain of an element and the image of one element under another. Both of these are abstractions and generalizations of the notions of domain of a function and image of a set under a function. Also the closed element of C-R algebra can be considered a generalization of the notion of continuous function. (Received March 4, 1954.)

552t. J. H. Curtiss: A generalization of the method of conjugate gradients for solving systems of linear algebraic equations.

In the description that follows, all matrices are real and $n \times n$, all vectors are real and $n$-dimensional, and a prime denotes transposition. Let $A$ be the matrix of the system to be solved; we assume that $A$ is arbitrary but nonsingular. Let $B$ be an arbitrary nonsingular matrix and let $T$ be a positive definite matrix. The generalization in question consists of an algorithm for generating a sequence of scalars $a_{0}, a_{1}, \cdots$ and a sequence of $B A T A^{\prime} B^{\prime}$-orthgoonal vectors $\delta_{0}, \delta_{1}, \cdots$ such that the solution of the given system can be expressed as a linear combination of the first $n$ vectors $\delta_{N}$ with coefficients given by the scalars $a_{N}$. The absolute value of the determinant of the system is given by $\left(a_{0} a_{1} \cdots a_{n-1} \operatorname{det} T\right)^{-1 / 2}|B|^{-1}$. The generalization is specialized in various ways to yield practical results. In particular it is shown that the specialization $B=I, T=I$ leads to a certain conjugate gradient algorithm proposed in his MIT doctoral thesis by E. J. Craig for the case of an arbitrary $A$, which seems to be simpler than that proposed by Hestenes and Stiefel (N.B.S. Journ. Res. vol. 49 (1952) pp. 409-436) for this case. (Received February 19, 1954.)

553. L. A. Henkin: Metamathematical theorems equivalent to the prime ideal theorems for Boolean algebras. Preliminary report. 
For notations see abstracts 562 and 564 . The following three statements $M_{1}, M_{2}, M_{3}$ are equivalent to each of $B_{1}-B_{5} . M_{1}$ : Let II be a set of formulas of sentential logic containing arbitrarily (possibly nondenumerably) many sentential symbols. If $I I$ is formally consistent, then truth-values can be assigned to the symbols so that all elements of $I I$ become true. $M_{2}$ : Let $\Sigma$ be a set of first-order sentences with arbitrarily many nonlogical constants. If $\Sigma$ is consistent, then it has a model. $M_{3}$ : Let $\Sigma$ be as in $M_{2}$. If $\mathfrak{S}$ is a system formed by a nonempty set $A$ and by finitary relations and operations on $A$, and if every finite subsystem of $S$ can be imbedded in a model of $\Sigma$, then $\subseteq$ can be imbedded in a model of $\Sigma$. Using the equivalence of $M_{1}-M_{8}$ with $B_{1}-B_{6}$, further purely mathemarical results are obtained. Thus, $B_{5}$ proves to be equivalent to the following statement $B_{6}$ (a sharper form of the representation theorem): $B_{6}$. Every Boolean algebra $\mathfrak{B}$ is isomorphic to a set algebra formed by subsets of a set which has the same power as $\mathfrak{B}$. Furthermore $B_{5}$ implies the following ordering principle: $O_{1}$. Every set can be simply ordered. Hence $a$ fortiori $B_{5}$ implies $A C$ for families of finite sets (a result previously obtained in a different way by Anne Davis). (Received March 8, 1954.)

554. Edwin Hewitt (p) and H. S. Zuckerman: Finite-dimensional convolution algebras.

Let $G$ be a finite semi-group (i.e., a set with an associative operation, written as $x y$ for $x, y \in G$; the existence of a unit is not assumed). The set $\mathcal{L}_{1}(G)$ consisting of all

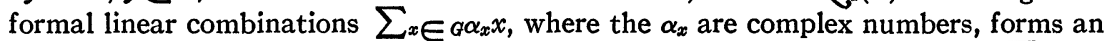
associative algebra over the complex number field under the operations $\left(\sum_{x} \alpha_{x} x\right)$ $+\left(\sum_{x} \beta_{x} x\right)=\sum_{x}\left(\alpha_{x}+\beta_{x}\right) x, \lambda\left(\sum_{x} \alpha_{x} x\right)=\sum_{x}\left(\lambda \alpha_{x}\right) x,\left(\sum_{x} \alpha_{x} x\right)\left(\sum_{y} \beta_{y} y\right)=\sum_{x} \sum_{y} \alpha_{x} \beta_{y} x y$. The following theorems are proved. I. $\mathcal{L}_{1}(G)$ is not a simple algebra if the order of $G=o(G)>1$. II. If $G$ is Abelian, then $\mathcal{L}_{1}(G)$ is semisimple if and only if, for every $x \in G$, there exists an integer $k>0$ such that $x^{k+1}=x$. III. The radical of $\mathcal{L}_{1}(G)$ may have any dimension $<o(G)$, but never $=o(G)$. IV. If $x y=x z$ implies $y=z$, for all $x, y, z \in G$, then $\mathcal{L}_{1}(G)$ is the Kronecker product of a group algebra and an algebra of all complex matrices $\left(a_{i j}\right)_{i, j=1}^{m}$ for which $a_{i j}=0$ for $i>1$. The noncommutative semisimple case is discussed. All $\mathcal{L}_{1}(G)$ are identified for $o(G)=2$ and 3. (Received March 15,1954 .)

555. R. D. James and Ivan Niven ( $)$ : Unique factorization in multiplicative systems.

In discussing unique factorization various writers cited certain multiplicative systems of positive integers having multiple factorings. For example, in the multiplicative system of all positive integers of the form $1+7 k$, the number 792 can be factored as $22 \cdot 36$ and also as $8 \cdot 99$. We examine all multiplicative systems composed of arithmetic progressions and decide the question of unique factorization. For a fixed positive integer $n$, let $M$ be a set of positive integers which are closed under multiplication, and such that if $x \in M$ and $y=x(\bmod n)$ with $y>0$, then $y \in M$. There is no loss of generality in assuming that $n$ is the smallest integer which can be used to define the set $M$. We prove that $M$ has the property of unique factorization if and only if $M$ consists of all positive integers relatively prime to $n$. (Received March 3,1954.)

\section{6t. J. H. McKay: Group extensions by left loops.}

If $F$ is a subgroup of a group $G$, any set of representatives for the right cosets of $F$ in $G$ is a left loop (binary operation, two-sided identity, unique solutions on the left) under a natural operation. In this paper the procedure for finding all extensions 
of a group $F$ by a left loop $R$ is separated into two parts by using the fact that any extension $G$ of $F$ by $R$ is homomorphic to a transitive permutation group on the elements of $R$ such that the kernel of the homomorphism is the largest subgroup of $F$ which is normal in $G$. In the first part (called a simple extension), this homomorphism is an isomorphism, while the other part is a Schreier extension. The set of all groups which can be simply extended by a given left loop is characterized. A definition is given for equivalence of left loops and its relationship to extensions is shown. Also, it is shown that the classes of equivalent left loops of degree $n$ are in 1-1 correspondence with the transitive permutation groups of degree $n$. (Received March 10,1954.)

557. Rimhak Ree and R. J. Wisner (p): A note on torsion-free nil groups.

A nil group $(B)$ is an (additively written) abelian group such that every ring $\Re$ with $B$ as its additive group has trivial multiplication, i.e., $A B=0$ for every $A, B \in \Re$. Szele [Zur Theorie der Zeroringe, Math. Ann. vol. 121 (1949)] completely characterized torsion nil groups and showed that there are no mixed nil groups. The question of the structure of torsion-free nil groups is considered here. Using a characterization of the additive rationals due to Beaumont and Zuckerman [Pacific Journal of Mathematics vol. 1 (1951) pp. 169-177], one finds sufficient conditions for torsion-free groups to be nil. The construction of examples of nil groups of arbitrary finite rank can be obtained easily. (Received March 11, 1954.)

558. Herman Rubin ( $\mathrm{p}$ ) and Dana Scott: Some topological theorems equivalent to the Boolean prime ideal theorem. Preliminary report.

For notation see abstracts 562-564 of Tarski. The following five theorems are equivalent to the prime ideal theorem $B_{3}$ for Boolean algebras: $T_{1}$. Tychonoff's compactness theorem for products of Hausdorff spaces. $T_{2}$. Alaoglu's theorem that the unit sphere of the adjoint of a Banach space is a compact Hausdorff space (cf. Ann. of Math. vol. $41(1940)$ pp. 252-267.) $T_{3}$. The Stone-Cech compactification theorem. $T_{4}$. The theorem that every complete and totally bounded uniform space is compact. $T_{5}$. Alexander's lemma that a space is compact if there is a subbase for the open sets enjoying the Heine-Borel property. (Cf. Proc. Nat. Acad. Sci. U.S.A. vol. 25 (1939) pp. 296298.) Each of $T_{1}-T_{5}$ implies that products of nonempty compact Hausdorff spaces are nonempty, and hence products of nonempty finite sets or nonempty closed subsets of a given compact Hausdorff space are nonempty. That the full Tychonoff theorem implies $A C$ was shown by Kelley (cf. Fund. Math. vol. 37 (1950) pp. 75-76) who also raised the question as to whether $T_{5}$ is equivalent to $A C$. In view of the above results it appears a positive answer to this question is possible only if $B_{3}$ implies $A C$. (Received March 8, 1954.)

\section{R. L. San Soucie: Right alternative rings of characteristic two.}

Let $F$ be the free nonassociative ring generated by $x_{1}$ and $x_{2}$ and suppose $R$ is any right alternative ring. Elements $t, u, v$ in $R$ form an alternative triple (Kleinfeld, Proc. Amer. Math. Soc. vol. 4 (1953) pp. 939-944) if (i) there exist elements $\alpha\left[x_{1}, x_{2}\right]$, $\beta\left[x_{1}, x_{2}\right], \gamma\left[x_{1}, x_{2}\right]$ in $F$ and elements $r_{1}, r_{2}$ in $R$ such that $t=\alpha\left[r_{1}, r_{2}\right], u=\beta\left[r_{1}, r_{2}\right]$, $v=\gamma\left[r_{1}, r_{2}\right]$ and (ii) if $s_{1}$ and $s_{2}$ are elements from an arbitrary alternative ring, and if $t^{\prime}=\alpha\left[s_{1}, s_{2}\right], u^{\prime}=\beta\left[s_{1}, s_{2}\right], v^{\prime}=\gamma\left[s_{1}, s_{2}\right]$, then $\left(t^{\prime}, u^{\prime}, v^{\prime}\right)=0 . R$ is said to have property (P) if $t, u, v$ an alternative triple in $R$ and $(t, u, v)^{2}=0$ imply $(t, u, v)=0$. Kleinfeld (loc. cit.) proves that a right alternative ring of characteristic not two having property $(P)$ is alternative, thus generalizing a theorem of Skornyakov that a right alter- 
native division ring of characteristic not two is alternative. The author has generalized the Skornyakov theorem to the characteristic two case (Bull. Amer. Math. Soc. Abstract 59-4-451). This paper provides a generalization of the Kleinfeld result by proving that a right alternative ring $R$ of characteristic two having property $(\mathrm{P})$ is alternative if and only if $R$ satisfies the identity $w(x y \cdot x)=(w x \cdot y) x$ for all $w, x, y$ in $R$. (Received February 26, 1954.)

560. Dana Scott: Prime ideal theorems for rings, lattices, and Boolean algebras. Preliminary report.

For notation see abstracts 562-564 of Tarski. The prime ideal theorem $B_{3}$ for Boolean algebras proves to be equivalent to each of the following statements $P_{1}$ and $P_{2}$. (Each of these comprehends $B_{3}$ as a particular case and seems to be much stronger than $B_{3}$.) $P_{1}$. In every commutative ring with unit, every proper ideal is included in some prime ideal. $P_{2}$. Let $\langle L, \cup, \cap\rangle$ be a lattice with unit 1 . In order that every proper ideal of this lattice be included in some prime ideal, it is necessary and suffcient that, for any elements $x, y, z$ in $L$, the formulas $x \cup_{y=1}$ and $x \cup_{z}=1$ imply $x \cup(y \bigcap z)=1$. Consider the following statements. $P_{1}^{\prime}:$ In every commutative ring with unit every proper ideal is included in some maximal ideal. $P_{2}^{\prime}:$ In every lattice with unit every proper ideal is included in some maximal ideal. $P_{2}^{\prime}$ is known to be equivalent to $A C$ (cf. the author's abstract 60-1-172). It is not known whether $P_{1}^{\prime}$ is equivalent either to $P_{1}$ or to $A C$. (Received March 8, 1954.)

\section{J. D. Swift: Sets of covering congruences.}

A covering congruence is defined as a finite set of ordered pairs $\left(r_{i}, m_{i}\right)$ such that $m_{i}>1$ for all $i ; m_{i} \neq m_{j}, i \neq j$; and such that any integer $n$ satisfies (at least) one congruence of the form $n \equiv r_{i}\left(\bmod m_{i}\right)$. If $\min m_{i}=M$, one says that the covering congruence is of order $M$. Sets up to order 6 are known. Infinite classes of covering congruences for small $M$ may be given of which the simplest is: Let $p$ be an arbitrary odd prime, $g$ an arbitrary singly even primitive root of $p$, then the set $\left(g^{k-1}, 2^{k}\right),\left(g^{k-1}\right.$, $\left.2^{k-1} p\right),\left(0,2^{p-1} p\right), 1 \leqq k \leqq p-1$, is a covering congruence. As a corollary, we may deduce: The sum of the reciprocals of the moduli of a covering congruence may be less than $1+\epsilon$ for any positive $\epsilon . M$ may be increased in various ways beginning with these sets but they do not appear to lead directly to an answer to the question: Does a covering congruence exist for arbitrary $M$ ? (Received March 10,1954.)

\section{Alfred Tarski: Prime ideal theorems for Boolean algebras and the axiom of choice. Preliminary report.}

A statement $S$ is said here to imply a statement $T$ if $T$ is derivable from $S$ by means of usual set-theoretical axioms excluding $A C$ (the axiom of choice). $S$ and $T$ are equivalent if $S$ implies $T$ and $T$ implies $S$. Boolean algebras are regarded as systems with two operations yielding joins and meets. Set algebras are Boolean algebras where joins coincide with unions, and meets with intersections. The following statements $B_{1}-B_{\mathfrak{5}}$ are mutually equivalent: $B_{1}$. Every Boolean algebra (with $0 \neq 1$ ) has a prime ideal. $B_{2}$. Every infinite Boolean algebra has a non-principal prime ideal. $B_{3}$. In every Boolean algebra every proper ideal is included in some prime ideal. $B_{4}$. In every Boolean algebra every principal ideal is isomorphic to a subalgebra containing $1 . B_{5}$. Every Boolean algebra is isomorphic to a set algebra. The equivalence of $B_{1}, B_{3}, B_{6}$ is a known result of Stone. As is known, $A C$ implies $B_{1}-B_{5}$. The problem is open whether the converse holds. However, $B_{1}-B_{5}$ cannot be proved without using $A C$. For Anne 
Davis showed (1948) that $B_{3}$ implies $A C$ restricted to families of finite sets; and (by results of Mostowski) $A C$ thus restricted is still not derivable from remaining settheoretical axioms. (Received March 8, 1954.)

563t. Alfred Tarski: Prime ideal theorems for set algebras and ordering principles. Preliminary report.

For notation see abstracts $562,564,553$. By a result of Henkin, $B_{1}-B_{5}$ imply the ordering principle $O_{1}$. E. W. Beth and the author found a purely mathematical proof of this implication by deriving $O_{1}$ directly from $S_{3}$. Using a similar method the author showed that $S_{3}$ implies two further ordering principles, $\mathrm{O}_{2}$ and $\mathrm{O}_{3}$ (of algebraic character): $\mathrm{O}_{2}$. Every Abelian group $\langle G,+\rangle$ in which all nonzero elements are of infinite order can be simply ordered; i.e., there is a relation $\leqq$ which simply orders $G$ and under which + is monotonic. $O_{3}$. Every field $\langle F,+, \cdot\rangle$ in which -1 is not a sum of squares can be simply ordered. Scott showed that $S_{3}$ implies the following: $O_{4}$. Every partial ordering of a set $A$ can be extended to a simple ordering of $A . O_{2}-O_{4}$ were originally established by means of $A C$. For $O_{2}$ see Tarski, Ann. Soc. Pol. Math. vol. 7 (1929) p. 267 ff.; for $O_{3}$ see Artin and Schreier, Abh. Math. Sem. Hamburg vol. 5 (1926) pp. 83-115; for $O_{4}$ see Szpilrajn, Fund. Math. vol. 16 (1930) pp. 386 ff. The problem remains open whether any of the statements $O_{1}-O_{4}$ is equivalent to $S_{3}$. (Received March 8, 1954.)

564t. Alfred Tarski: Prime ideal theorems for set algebras and the axiom of choice. Preliminary report.

For notation see abstract 562. A set algebra is called complete if it is formed by all subsets of a certain set. Let $S_{1}-S_{4}$ be respectively the statements $B_{1}-B_{4}$ restricted to complete set algebras. Let $S_{5}$ be $B_{5}$ restricted to those Boolean algebras which are homomorphic images of complete set algebras. $S_{1}$ and $S_{4}$ are obviously provable without using $A C$. The author showed that $S_{3}$ and $S_{5}$ are both equivalent to $B_{1}-B_{5}$ (and hence cannot be proved without using $A C$ ). Scott noticed that, by analyzing the proof of this equivalence, the following can be established without applying $A C$ : Every Boolean algebra is a homomorphic image of a set algebra. $S_{3}$ implies $S_{2}$; whether the converse holds is not known. It is unlikely that $S_{2}$ is independent of $A C$, for (by a result of Sierpinski) $S_{2}$ implies the existence of sets non-measurable $(L) . S_{1}-S_{3}$ are equivalent to certain measure-theoretical statements; e.g., $S_{3}$ is equivalent to the following: Let $\mathcal{Z}$ be a family of sets, $X \subseteq A$ such that $A$ is not a union of finitely many sets $X \in \mathcal{F}$; then there is a two-valued finitely additive measure $m$ on all sets $X \subseteq A$ such that $m(A)=1$ and $m(X)=0$ for every $X \in \mathcal{F}$. (Received March 8, 1954.)

565. Morgan Ward: Mappings of the positive integers into themselves which preserve division.

Let $\phi: \phi(n)$ denote any mapping of the integers $0,1,2, \cdots$, into themselves such that $\phi(n)=0$ if and only if $n=0 ; \phi(1)=1 ; \phi(n)$ divides $\phi(m)$ if $n$ divides $m$. Necessary and sufficient conditions are given for $\phi$ to admit a rank function $\rho: \rho(m)$ such that for every modulus $m, \phi(n) \equiv 0(\bmod m)$ if and only if $n \equiv 0 \bmod \rho(m)$. The results generalize and extend from properties of the Lucas numbers $\left(\alpha^{n}-\beta^{n}\right) /(\alpha-\beta)$. (Received April 6, 1954.)

566. P. S. Wolfe: Some games of infinite length. 
Gale and Stewart (Infinite games of perfect information, Contributions to the theory of games II, Princeton, 1953, pp. 245-266) have considered zero-sum two-person games with no umpire moves having no stop rule, and ask whether such a game is strictly determined in pure strategies when the payoff is the characteristic function of a set of plays which is a $G_{\delta}$ in a certain topology. An affirmative answer is given by proving the strict determinateness of a related game in which the payoff from the play $s$ is 1 when an infinite number of the positions constituting $s$ belong to a certain subset of positions, and 0 otherwise. This result yields the strict determinateness of the two games having payoffs lim $\sup _{i \rightarrow \infty} h(s(i))$ and $\alpha \sup _{i} h(s(i))+\beta \inf _{i} h(s(i))$, where $h$ is any bounded function of positions and $s(i)$ is the $i$ th position in the play $s$. (Received March 18, 1954.)

\section{AnAlysis}

567. H. J. Bremermann: Complex convexity. I. Regions of holomorphy.

Let $z$ denote the point $\left(z_{1}, \cdots, z_{n}\right)$ in the space of $n$ complex variables $C^{n}$, and let $\delta_{D}(z)$ denote the Euclidean distance of the point $z \in D$ from the boundary of $D\left(D \subset C^{n}\right)$. A region of holomorphy $H$ is a region such that there exists a function holomorphic in $H$ but singular at every boundary point. Let $H$ be a region of holomorphy, let $S, T$ be point sets, $S \cup T \subset \subset H$, such that for every function $h(z)$, holomorphic in $H$, $\max _{z \in S \cup T}|h(z)|=\max _{z} \in T|h(z)|$. Let $\chi(z)$ be a fixed function, holomorphic and different from zero in $H$. Then $\min _{z \in S \cup T}|\chi(z)| \delta_{H}(z)=\min _{z \in T}|\chi(z)| \delta_{H}(z)$. Using this theorem it is easy to derive the holomorph-convexity of Cartan-Thullen as well as certain new results concerning the holomorph convexity. Also a new and simpler proof of the following theorem is given: If $H$ is a region of holomorphy, $-\log \delta_{H}(z)$ is a plurisubharmonic function in $H$. The function - $\log \delta_{H}(z)$, being plurisubharmonic, generates a positive semidefinite metric form: $d s^{2}=\sum_{\mu, \nu=1}^{n}\left(\partial^{2}\left(-\log \delta_{H}(z)\right) / \partial z_{\mu} \partial \bar{z}_{\nu}\right), d z_{\mu} d \bar{z}_{\nu}$. Other metrics and distance functions are studied and compared with the Bergman kernel function and the holomorphically invariant metric form generated by it. (Received May 1, 1954.)

568t. H. J. Bremermann: Complex convexity. II. Pseudo-convex regions.

A region $D \subset C^{n}$ which has the property that $-\log \delta_{D}(z)$ is plurisubharmonic in $D$ is called "pseudo-convex." Thus any region of holomorphy is pseudo-convex. (The converse has also been proved by the author.) It is shown that the notions "pseudoconvex region" and "plurisubharmonic function" are an extension of the notions "convex region" and "convex function" from the real space $R^{n}$ to complex space $C^{n}$ (in the same sense as the notion "Hermitian form" is an extension of the notion "quadratic form" from the $R^{n}$ to $C^{n}$ ). Also, by associating with every convex region a pseudoconvex tube region and with every convex function a plurisubharmonic function defined in a tube region, it is proved that any theorem that is true for all pseudo-convex regions and for all plurisubharmonic functions is in a corresponding form true for all convex regions and convex functions. (Received May 1,1954.)

569t. H. J. Bremermann: Complex convexity. III. Bergman kernels and other kernel functions.

The correspondence theorem in II leads the author to consider several complex variables methods in the convex case. In particular, he interprets the Bergman kernel 
function and invariant metric in the convex case. It is also shown that the non-negative convex functions as well as the non-negative plurisubharmonic functions form a Hilbert space with a reproducing kernel, if one introduces the inner product $(f, f)$ $=\int_{D} f^{2} d \omega$ ( $d \omega$ the volume element). The existence of such a kernel follows immediately from the fact that the inequality $f^{2}\left(x^{(0)}\right) \leqq \nu_{n}(r)^{-1} \int_{\mid x-x(0)} \mid<r f^{2} d \omega_{n}$ holds in the convex case $\left(\nu_{n}(r)\right.$ the volume of the $n$-sphere), and the corresponding inequality $f^{2}\left(z^{(0)}\right)$ $\leqq \nu_{2 n}(r)^{-1} \int_{\mid \varepsilon-r(0)}\left({ }_{r} f^{2} d \omega_{2 n}\right.$ holds in the pseudo-convex case. Finally, he finds the analogue to "Bergman's extended class of functions" in the convex case, and by reversing the method comes to define a different extended class for (pseudo-convex) analytic polyhedra in the $C^{n}$. (Received May 1, 1954.)

570. M. A. Dengler: Laplace transforms related to the theory of transverse waves in elasticity.

The object of the investigation is the derivation of certain Laplace transforms which are directly related to the theory of transverse waves in elastic media as deduced by Goland and the author in Transverse impact of long beams, including rotatory inertia and shear effects, Proceedings of the First U. S. National Congress for Applied Mechanics, Chicago, 1950. The theory referred to here is based on the Timoshenko bending mechanism which, as well known, accounts for the influences of rotatory inertia and transverse shear and therefore permits the calculation of the transient response of elastic structures with satisfactory accuracy. The Laplace transforms presently reported must be considered as the central domain of these theoretical studies, when seen from the mathematical point of view. Relations between transforms and inverse transforms are derived by contour integrations and appear in closed and real form. (Received March 1, 1954.)

\section{Solomon Leader: Convergence topologies for measures.}

In the norm topology for signed measures there may exist functionals which cannot be represented as integrals in the original space. This defect is removed by introducing weaker topologies defined by convergence of nets, so that functionals can be expressed as integrals and convergence of positive measures is equivalent to weak convergence. The space $S$ of real-valued, additive functions on a Boolean algebra of sets with the topology of simple convergence $\left[F_{\alpha} \rightarrow 0\right.$ if $F_{\alpha}(E) \rightarrow 0$ for each $E$ ] has as dual space all step functions. The space $B$ of bounded functions in $S$ with the topology of bounded convergence $\left[F_{\alpha} \rightarrow 0\right.$ if $F_{\alpha}(E) \rightarrow 0$ and $\lim \sup _{\alpha}\left\|F_{\alpha}\right\|$ is finite] has as dual space uniform limits of step functions. Similar results hold for countably additive measures. The space $R$ of bounded, regular, signed measures on a normal space with the topology of regular convergence [for positive measures, $F_{\alpha} \rightarrow F$ if $F_{\alpha}(E) \rightarrow F(E)$ wherever $F($ bdy $E)=0$ ] has as dual space bounded, continuous functions. Continuous operators are characterized through the invariance of the dual space under the adjoint operators. This gives an existence theorem for transition probabilities generating a stochastic semigroup. This work was supported by the Research Council of Rutgers University. (Received March 1, 1954.)

\section{2t. A. R. Lovaglia: Locally uniformly convex Banach spaces.}

In this paper a normed linear space is defined to be locally uniformly convex (l.u.c.) if given $\epsilon>0$ and an element $x$ with $\|x\|=1$, there exists $\delta(\epsilon, x)>0$ such that $\|x+y\| / 2 \leqq 1-\delta(\epsilon, x)$ whenever $\|x-y\| \geqq \epsilon$ and $\|y\|=1$. Let $\left\{B_{n}\right\}$ be a sequence of 1.u.c. Banach spaces. Let $P_{2}\left(B_{n}\right)$ be the space of all sequences $x=\left\{x^{n}\right\}, x^{n} \in B_{n}$, for 
which $\sum_{n-1}^{\infty}\left\|x^{n}\right\|_{n}^{2}$ is convergent. $\left(\|\|_{n}\right.$ is the norm in $B_{n}$.) Let $\|x\|=\left(\sum_{n-1}^{\infty}\left\|x^{n}\right\|_{n}^{2}\right)^{1 / 2}$. Then $P_{2}\left(B_{n}\right)$ is a 1.u.c. Banach space. It is shown that there exist l.u.c. Banach spaces not isomorphic to any uniformly convex Banach space. (J.A. Clarkson, Uniformly convex spaces, Trans. Amer. Math. Soc. vol. 40 (1936) pp. 396-414.) From this it follows that the notion of 1.u.c. is essentially different from M. M. Day's notion of local uniform convexity near a point $b_{0}$. (M. M. Day, Uniform convexity, Bull. Amer. Math. Soc. vol. 49 (1943) pp. 745-750.) If $B$ is a 1.u.c. Banach space and linear functionals attain their maximum on the unit sphere, then the norm in the adjoint space $\bar{B}$ is strongly differentiable. (V. Smulian, Sur la dérivabilité de la norme dans l'espace de Banach, C. R. (Doklady) Acad. Sci. URSS vol. 27 (1940) pp. 643-648.) If the adjoint space $\bar{B}$ is l.u.c. then the norm in $B$ is strongly differentiable. If $B$ has a basis $\left\{x_{i}\right\}$ with the property that for each number $c>0$ there exists a number $r_{0}>0$ such that $\left\|\sum_{i=1}^{n} a_{i} x_{i}\right\|=1$ and $\left\|\sum_{i=n+1}^{\infty} a_{i} x_{i}\right\| \geqq c$ imply $\left\|\sum_{i=1}^{\infty} a_{i} x_{i}\right\| \geqq 1+r_{c}$, then $B$ is isomorphic to a l.u.c. space. (Received March 18, 1954.)

\section{Michael Maschler: On properties of minimal domains.}

By using the method of the kernel function, S. Bergman introduces the mapping of a domain onto a "minimal domain." A minimal domain $D$ in the $z$-plane, with respect to $z=t \in D$, is a bounded domain such that any function $f(z)\left[f(t)=0, f^{\prime}(t)=1\right]$, regular in $D$, maps it onto a domain whose area is not smaller than the area of $D$. While every simply-connected minimal domain is a circle, a multiply-connected minimal domain is in general, according to an unpublished result of $M$. Schiffer, non-schlicht. A minimal domain is defined in the space of $n$ complex variables in a similar way. The results stated below are special cases of analogous theorems in that space: A necessary and sufficient condition for a domain $D$ to be a minimal domain w.r.t. $t$ is that its Bergman's kernel function $K_{D}(z, t)=$ constant. This constant must be $1 / S$ where $S$ is the area of $D$. For a minimal domain $D$ w.r.t. $t$, and only for such a domain, $S \cdot f(t)=\iint_{D} f(z) d x d y(z=x+i y)$, for every $f(z) \in L^{2}(D)$. These results lead to the determination of various properties of minimal domains and have applications in the theory of conformal and pseudo conformal transformations. (Received March 10, 1954.)

\section{E. G. Straus: On integer-valued entire functions.}

It is shown that an entire function $f(z)$ which satisfies $f(z)=O\left(e^{c|z|^{m-1}}\right)$, and all whose derivatives at $m$ points $z_{1}, \cdots, z_{m}$ are rational integers, must satisfy a linear differential equation with integral constant coefficients. More precisely, if one sets $z_{1}=0$, then $f(z)$ is either (i) a polynomial with rational coefficients, or (ii) $\sum c_{k} e^{k z}$, $c_{k}$ rational, or (iii) $\sum\left(c_{k} \exp \left(k m^{1 / 2} z\right)+\bar{c}_{k} \exp \left(-k m^{1 / 2} z\right)\right)$ where $m$ is a rational integer and $c_{k}, \bar{c}_{k}$ are conjugate elements of $R\left(m^{1 / 2}\right)$. On the other hand there is a continuum of entire functions of any order $\rho \geqq m$ which have integral derivatives of all orders at $m$ prescribed points. Generalizations, such as replacing "rational integer" by "algebraic integer" or even "algebraic number" are possible. (Received May 1, 1954.)

\section{Applied Mathematics}

575. K. O. Friedrichs and D. H. Hyers ( $\mathrm{p})$ : The existence of solitary waves.

Recently M. Lavrentieff (Akad. Nauk Ukrain RSR Sbirnik Prac. Inst. Mat. vol. 1946 no. 8 (1947) pp. 13-69; cf. Math. Revs. vol. 14, p. 102) has given a proof of the existence of two-dimensional solitary gravity waves of permanent type by considering 
them as limiting cases of periodic waves as the wavelength tends to infinity. In the present paper their existence is proved in a direct manner. Introducing the dimensionless parameter $\gamma=g h U^{-2}$ where $g$ is the acceleration of gravity, $h$ the depth of liquid at infinity, and $U$ the velocity of propagation, it is shown that for each value of $\gamma<1$ and sufficiently close to one, a symmetric solitary wave exists. A decisive step in the method is the introduction of new independent variables involving a stretch in one direction, analogous to the change of variables used by Friedrichs in his derivation of the shallow water theory (Com. Pure and Appl. Math. vol. 1, p. 81) and by Keller in his approximate treatment of solitary and periodic waves (ibid., vol. 1, p. 323). Thus, in a sense, the result affords a justification of the second order shallow water theory for this problem. (Received March 10, 1954.)

\section{6t. C. H. Papas: An application of Sommerfeld's complex order wave functions to antenna theory.}

In the past wave functions of integral order have been used advantageously in the solution of boundary-value problems. However, in some instances these wave functions are completely alien to the problem and introduce difficulties which, indeed, can be resolved but only at the expense of logical simplicity. To place in evidence the usefulness and the "naturalness" of complex order wave functions (A. Sommerfeld, Partial differential equations, New York, Academic Press, 1949), the input impedance of a boss antenna is examined theoretically with the aid of these functions. Following Schwinger's formalism (H. Levine and C. H. Papas, Journal of Applied Physics vol. 22 , pp. 29-43) the input impedance is cast in variational form. This form involves a Green's function and an aperture electric field, both of which are expressed in terms of complex order wave functions. A physically plausible choice of trial functions yields a rather neat expression for the input impedance. (Received January 18, 1954.)

577t. Gabor Szegö: Inequalities for certain eigenvalues of a membrane of given area.

Let the domain $D$ with an analytic boundary curve $C$ represent the membrane in its position of equilibrium. We denote by $\lambda_{1}$ the least positive eigenvalue of $D$ with boundary condition $u=0$ and by $\mu_{2}$ its least positive eigenvalue with boundary condition $\partial u / \partial n=0$. If $A$ is the area of $D$, we have the Rayleigh-Faber-Krahn inequality $\lambda_{1} \geqq j(A / \pi)^{-1 / 2}$ where $j=2.40$ is the least positive zero of $J_{0}(x)$. In the present paper an inequality of opposite nature is proved for $\mu_{2}$ which was announced without proof by E. T. Kornhauser-I. Stakgold (Journal of Mathematics and Physics vol. 31 (1952) p. 45). This is the following: $\mu_{2} \leqq p(A / \pi)^{-1 / 2}$ where $p=1.84$ is the least positive zero of $J_{1}^{\prime}(x)$. In the remaining part of the paper the next two eigenvalues $\lambda_{2}, \lambda_{3}$ and $\mu_{2}, \mu_{3}$ for the boundary problems mentioned above are evaluated in a certain approximate sense provided $C$ is a "nearly circular" curve. (Received February 4,1954 .)

578. W. R. Wasow: Discrete approximations to elliptic differential equations.

The method of Collatz [Zeitschrift für Angewandte Mathematik und Mechanik vol. 13 (1933) pp. 56-57] for the appraisal of the truncation error in the solution of boundary value problems for elliptic differential equations by means of finite differences is generalized so as to apply to general types of nets and to a general class of interpolation schemes at curved boundaries. Following Petrovsky the whole class of discrete approximations considered can be treated in a unified fashion by using 
Stieltjes integral notation. The theory applies also to the discretization error connected with solutions of boundary value problems by random walks that are not restricted to the points of any net. With the help of the results obtained the extrapolation technique of L. F. Richardson [Phil. Trans. (A) vol. 210 (1911) pp. 307-357] for the improvement of approximations obtained by finite difference methods can be analyzed mathematically. It turns out that this method is applicable to problems in domains with curved boundaries only if the boundary values are interpolated by a formula so precise that the resulting contribution to the truncation error is small of higher order than the contribution originating in the interior of the domain. (Received February 16, 1954.)

\section{LOGIC AND Foundations}

\section{Chen-Chung Chang: $A$ remark on convex classes.}

For notation see Tarski, Abstract 59-4-500. A class $K$ of non-empty algebraic systems (with operations and relations) is called (i) convex, or (ii) weakly convex, if for every $\mathfrak{A}, \mathfrak{B}, \mathfrak{E} \in K$, such that $\mathfrak{B}$, $\mathfrak{C}$ are subsystems of $\mathfrak{A}$, (i) the intersection $\mathfrak{B} \cap \mathfrak{C}$ belongs to $K$, or (ii) $\mathfrak{B} \cap \mathfrak{E} \in K$ whenever $\mathfrak{B} \cap \mathfrak{C} \neq 0$. Theorems: I. If $K \in A C_{\delta}$ and $K$ is (i) convex, or (ii) weakly convex, then for any nonempty subclass $\mathcal{L}$ of $K$ consisting of subsystems of a system $\mathfrak{A} \in K$, (i) the intersection $\cap \mathcal{L}$ belongs to $K$, or (ii) $\cap \mathcal{L}$ $\in K$ whenever $\cap \mathcal{L} \neq 0$. II. If $K \in A C_{\delta}$ and $K$ is convex, or even weakly convex, then, for every non-empty subclass $\mathcal{L}$ of $K$ which is simply ordered by the subsystem relation, the union $\cup \mathcal{L}$ belongs to $K$. Theorems I and II remain true if the formula $K \in A C_{\delta}$ is replaced by the condition that $K$ is quasi-arithmetical in the sense of Henkin (Trans. Amer. Math. Soc. vol. 74, p. $418 \mathrm{ff}$.) or, more generally, pseudo-arithmetical in the sense of Tarski (Contributions to the theory of models, to appear in Proc. Dutch Acad.). Theorem II improves a result in Robinson, On the metamathematics of algebra, 1951, p. 117, 9.1.3., who uses a much stronger definition of convexity. (Received March 8, 1954.)

\section{0t. Roland Fraïssé: Some elementary properties of universal classes.}

Given two relational systems $\mathfrak{A}, \mathfrak{B}$, the formula $\mathfrak{B}<\mathfrak{A}$ means that $\mathfrak{B}$ can be isomorphically embedded in $\mathfrak{A}$. A relational system $\mathfrak{A}=\left\langle A, R_{1}, \cdots, R_{n}\right\rangle$ will be called finite if the set $A$ is finite. The following are proved (for notation, see Tarski, Bull. Amer. Math. Soc. Abstract 59-4-500): (1) A class $K$ of relational systems is a $U C$ (a $U C_{\delta}$ ) iff there exists a finite (a denumerable) set $\left\{\mathfrak{F}_{1}, \cdots, \mathfrak{F}_{m}, \cdots\right\}$ of finite relational systems, such that $\mathfrak{Y} \in K$ iff $\mathfrak{X} \ngtr \mathfrak{F}_{i}(i=1, \cdots, m)$. (2) One can always assume the $\mathfrak{F}_{i}$ 's are mutually incomparable $\left(\mathfrak{F}_{i} \nless \mathfrak{F}_{j}\right.$ when $\left.i \neq j\right)$; then the $\mathfrak{F}_{i}$ 's are all the finite relational systems (defined up to isomorphism) which do not belong to $K$ and such that every proper subsystem of each of them belongs to $K$. These $\widetilde{\mho}_{i}$ will be called associated with $K$. (3) If $K, L \in U C_{\delta}$, then $L \subseteq K$ iff, for every $\mathfrak{F}_{i}$ associated with $K$, there exists $\mathbb{S}_{j}<\mathfrak{F}_{i}\left(\mathfrak{S}_{j}\right.$ associated with $\left.L\right)$. (4) The formula $\mathfrak{B}<\mathfrak{A}$ means that every finite subsystem of $\mathfrak{B}$ can be isomorphically imbedded in $\mathfrak{A}$; it is equivalent to say that every $U C$ which contains $\mathfrak{A}$ contains also $\mathfrak{B}$. Assuming $K \in U C, K$ is indecomposable for $\cup$ (i.e. it is not any union of $U C$ 's different from $K$ ) iff there exists a relational system $\Re$ such that $\mathfrak{A} \in K$ iff $\mathfrak{A} \prec \Re$. (Received March 8, 1954.)

581. Roland Fraïssé: Some relational systems which generalize simply ordering relations.

A relational system $\mathfrak{A}=\left\langle A, R_{1}, \cdots, R_{n}\right\rangle$ will be called $p$-monotypic $(n, p$ natural 
numbers) if all subsystems of $\mathfrak{A}$ having $p$ elements are isomorphic. $\mathfrak{A}$ will be called monotypic if it is $p$-monotypic for every $p$ ( $A$ being infinite). The following are proved: (1) If, $A$ being infinite, $\mathfrak{A}$ is $p$-monotypic, then it is $q$-monotypic for $q<p$. This result fails if $A$ is finite. (2) If $\mathfrak{A}=\left\langle A, R_{1}, \cdots, R_{n}\right\rangle$ is monotypic, there exists an ordering (i.e. simply ordering) relation $I$ defined on $A$, such that $\left\langle A, R_{1}, \cdots, R_{n}, I\right\rangle$ is monotypic. (3) Let $I, J$ be two ordering relations such that $\mathfrak{B}=\left\langle A, R_{1}, \cdots, R_{n}, I\right\rangle$ and $\mathfrak{C}=\left\langle A, R_{1}, \cdots, R_{n}, J\right\rangle$ are monotypic. Either, for every $p$, the subsystems of $\mathfrak{B}$ and $(c$ having $p$ elements are all isomorphic, or this is true when $J$ is replaced by its converse. (4) If $\mathfrak{A}$ is monotypic and $I$ an ordering relation as in (2), then each relation $R_{i}(1 \leqq i \leqq n)$ is in the least class $K$ of relations, such that the identity relation, $I$, and $A^{k}(k=1,2, \cdots)$ are in $K$, and such that $K$ is closed under the following operations: complementation, formation of union of two elements, permutation of arguments, passing from an $m$-ary relation $R$ to an $(m+1)$-ary relation $R^{\prime}$ where $R^{\prime}\left(x_{1}, \cdots, x_{m}, x_{m+1}\right)$ holds iff $R\left(x_{1}, \cdots, x_{m}\right)$ holds. (Received March $8,1954$. )

582t. Herman Rubin and P. C. Suppes: $A$ note on two-place predicates and fitting sequences of measure functions.

The main purpose of this note is to show by means of a counterexample that Carnap's measure function $m^{*}$ (Logical foundations of probability) does not have the intuitively desirable property of fittingness when we consider finite languages using two-place predicates. Following Carnap, the notation: $1 m^{*}, 2 m^{*}, \cdots$ is used for the measure function $m^{*}$ in languages $\mathcal{L}_{1}, \mathcal{L}_{2}, \cdots$, where the only predicate in $\mathcal{L}_{i}$, for $i=1,2, \cdots$, is the two-place predicate ' $R$ '. Let ' $a$ ' and ' $b$ ' be two individual names and let $i$ be the sentence: $R a a \& R a b \& R b a \& R b b$. We easily verify that $2 m^{*}(i)=.1$, and with somewhat more trouble that $3 m^{*}(i)=23 / 312$. Hence the sequence $1 m^{*}$, $2 m^{*}, \cdots$ is not fitting. A group-theoretic theorem which guides the construction of such counterexamples is stated and proved, and is used in conjunction with results of Davis (Proc. Amer. Math. Soc. vol. 4 (1953) pp. 486-495) to show that $4 m^{*}(i)$ is neither of the previous values. (Received March 5,1954.)

\section{A. C. Sugar: A foundational analysis of Lagrange's equations.}

Beginning with a set theoretic definition of function and a modified form of Menger's notation the ambiguity of the conventional notation for functions is eliminated. Subsequently the question is raised as to what is meant in the standard derivations of Lagrange's equations by $\partial \dot{x} / \partial \dot{q}$ and $\partial \dot{x} / \partial q$, where $\dot{x}=f^{\prime}(q) \dot{q}$. Since the above notation indicates that $\dot{x}$ is to be differentiated, then " $\dot{x}$ " is being used to designate a function, say $F$. On the basis of the above indicated operation of partial differentiation, $F$ must be a function of more than one argument and therefore is not a function of time only. Furthermore $F$ cannot be a function of $q$ and $\dot{q}$ because these arguments are not independent as required by the definition of partial differentiation. These difficulties concerning $\dot{x}$ will appear of course also for kinetic energy. To illustrate (still using conventional notation) let the position $x=q^{2}$ and the generalized coordinate $q=e^{t}$; then the kinetic energy may, among others, be given by $T=2 m q^{2} \dot{q}^{2}$ $=2 m q^{4}=2 m \dot{q}^{4}$. It is interesting to ask what $\partial T / \partial q$ represents in this case. The physicist would take a "derivative" of the function defined by the first of the three expressions. A precise derivation of Lagrange's equations may be based on either of two points of view. We are either differentiating a generalized kinetic energy function or carrying out a process of symbolic partial differentiation of a specified kinetic energy function of two dependent arguments. (Received March 8, 1954.) 
584t. P. C. Suppes: Axiomatic analysis of the method of equalappearing intervals.

In this paper a set of independent axioms is given which characterizes the procedures of measurement that psychologists lump together under the title of the method of equal-appearing intervals. The axioms are based on three primitive notions: (1) a finite set $K$, ordinarily a set of tones or colors, or in the case of utility, economic goods; (2) a binary relation $\mathrm{P}$ of precedence whose field is $K$; and (3) a quaternary relation $\mathrm{E}$ whose fields is $K$ such that in the intended interpretation $x, y \mathrm{E} u, v$ only if the difference between $x$ and $y$ is equivalent to that between $u$ and $v$. The expected adequacy theorem is established, namely that: (A) there exists a realvalued function $\phi$ defined on $K$ such that (i) $x \mathrm{P} y$ if and only if $\phi(x)<\phi(y)$, and (ii) $x, y \mathrm{E} u, v$ if and only if $\phi(x)-\phi(y)=\phi(u)-\phi(v)$; (B) $\phi$ is unique up to a linear transformation. It is also proved that it is not possible to add further axioms based on these primitive notions so as to strengthen $(B)$ of the adequacy theorem to: $\left(B^{\prime}\right) \phi$ is unique up to a similarity transformation. Due to the assumption that $K$ is finite and that differences between adjacent elements are equivalent, the axiomatization and proof of adequacy given here are considerably simpler than that given for more general situations in Suppes and Winet (Bull. Amer. Math. Soc. Abstract 60-1-167). (Received March 8, 1954.)

\section{TOPOLOGY}

585t. C. E. Burgess: Some properties of certain types of homogeneous continua.

A continuum $M$ is said to be $n$-homogeneous if for any $n$ points $x_{1}, x_{2}, \cdots, x_{n}$ of $M$ and any $n$ points $y_{1}, y_{2}, \cdots, y_{n}$ of $M$ there is a homeomorphism of $M$ onto itself carrying $x_{1}+x_{2}+\cdots+x_{n}$ onto $y_{1}+y_{2}+\cdots+y_{n}$. In the author's paper Some theorems on n-homogeneous continua, Bull. Amer. Math. Soc. Abstract 60-3-309, it was shown that if $n>1$ and the bounded plane continuum $M$ is $n$-homogeneous, then $M$ is a simple closed curve. If this hypothesis is changed so that $M$ is an unbounded proper subcontinuum of the plane, then $M$ is homeomorphic with a straight line. The following theorems are also proved. (1) If every proper subcontinuum of the compact metric continuum $K$ is homogeneous, then $K$ is hereditarily indecomposable. (2) If $K$ is a decomposable bounded plane continuum such that for any two nondegenerate proper subcontinua $K_{1}$ and $K_{2}$ of $K$ there is a homeomorphism of $K$ onto itself carrying $K_{1}$ onto $K_{2}$, then $K$ is a simple closed curve. (Received March 1, 1954.)

\section{W. S. Miller: A non-Hausdorf, finest bicompact space.}

A space is constructed from the closed unit circle with the origin deleted, and two points $O$ and $O^{\prime}$. Neighborhoods of the points $O$ and $O^{\prime}$ are defined so that they have no disjoint neighborhoods, so that the space is bicompact, and such that every bicompact set in the space is closed. (Received March 10,1954.)

\section{7t. Barrett O'Neill: A comparison fixed point theorem.}

A topological space $X$ is called a Lefschetz space provided the Lefschetz theorem holds for $X$ in this sense: if $f$ is a self-map of $X$ for which the Lefschetz number exists and is nonzero, then $f$ has a fixed point. Let $X$ and $Y$ be compact spaces. Write 
$X<Y$ provided: given any element $U$ of the uniform structure on $X$ there is a homology equivalence, $f: X \rightarrow Y, g: Y \rightarrow X$, such that $g(f(x)) \in U(x)$ for all $x \in X$. This relation is an order relation on the class of compact spaces. This theorem is proved: if $X<Y$ and $Y$ is a Lefschetz space, then so is $X$. The criterion is applied to give examples of non-HLC spaces which are Lefschetz spaces and, in particular, have the fixed point property. (Received March 9, 1954.)

\section{J. W. GREen, Associate Secretary}

\section{RESEARCH PROBLEMS}

\section{Walter Rudin: Maximum modulus algebras.}

Let $D$ be a domain bounded by a simple closed curve $C$, and let $K=D \cup C$. In Duke Math. J. vol. 20 (1953) pp. 449-458, the following theorem is proved: Let $A$ be an algebra of complex-valued functions continuous on $K$ and suppose (1) for every $f \in A$ there is a point $z_{0} \in C$ such that $|f(z)| \leqq\left|f\left(z_{0}\right)\right|(z \in K)$; (2) $A$ contains a nonconstant function which is analytic in $D$; (3) $A$ contains a schlicht (that is, one-to-one) function. Then every $f \in A$ is analytic in $D$. Is the conclusion valid if (3) is omitted from the hypotheses? Is it possible to weaken (3), for instance by requiring that $A$ separates points? (Received April 6, 1954.)

20. Walter Rudin: Radial limits of analytic functions.

If $f$ is analytic in the interior $U$ of the unit circle, and if there exists a set $E$ of positive measure such that $f\left(r e^{i \theta}\right)$ is bounded for $0 \leqq r<1, \theta \in E$, does $\lim _{r \rightarrow 1} f\left(r e^{i \theta}\right)$ necessarily exist for almost all $\theta \in E$ ? The same question may be asked about functions meromorphic in $U$. ( Received April 6, 1954.) 